\title{
Long-term survival after resections for pancreatic ductal adenocarcinoma. Single centre study
}

\author{
Martin Lovecek ${ }^{\mathrm{a}}$, Pavel Skalicky ${ }^{\mathrm{b}}$, Dusan Klos ${ }^{\mathrm{b}}$, Linda Bebarova ${ }^{\mathrm{a}}$, Cestmir Neoral ${ }^{\mathrm{b}}$, Jiri Ehrmann Jr.c, Jana Zapletalovad, \\ Hana Svebisova ${ }^{\mathrm{e}}$, Radek Vrba ${ }^{\mathrm{b}}$, Martin Stasek ${ }^{\mathrm{a}}$, Tharani Yogeswara ${ }^{\mathrm{a}}$, Roman Havlik ${ }^{\mathrm{b}}$
}

Aim. To analyse the 5-year survival rate of patients undergoing radical surgery for pancreatic ductal adenocarcinoma (PDAC) and to identify prognostic factors.

Methods. A prospectively maintained database of 90 consecutive patients who underwent radical resection for PDAC was analysed. Survival was evaluated using the Kaplan-Meier method. Log-rank test and Cox regression analysis were used for the evaluation of prognostic factors. $P$ values less than 0.05 were considered significant.

Results. Mean age ( \pm standard deviation) was $63.2 \pm 8.6$ years (female $28.9 \%$ and male $71.1 \%$ ). Tumour localisation was in the head in 76 (84.5\%), multifocal in 3 (3.3\%) and in the body/tail in 11 (12.2\%). Pancreatic head resection was performed in 75 (83.3\%), total pancreatectomy in 4 (4.4\%) and distal pancreatectomy with splenectomy in 11 (12.2\%), with standard lymphadenectomy. Venous resection was in 4 (4.4\%). Thirty-day and in-hospital mortality occurred in 1 (1.1\%), 90-day mortality was 3.3\%. On univariate analysis absence of perineural and vascular invasion, stage, absence of lymph node infiltration and no need for transfusion were associated with improved overall survival. On multivariate analysis vascular invasion $\mathrm{HR}=3.137(95 \% \mathrm{Cl}$ : 1.692-5.816; $P=0.0003)$ and postoperative complications $\mathrm{HR}=2.004$ (95\%Cl: 1.198-3.354; $P=0.008$ ) were identified as significant independent predictors of survival. The five-year survival rate was $18.9 \%$, with five-year recurrence-free survival of $16.7 \%$.

Conclusion. Vascular invasion and postoperative complications were independent prognostic factors after curative resections of pancreatic cancer in studied cohort.

Key words: pancreatic ductal adenocarcinoma, long term survival, complications, surgical therapy

Received: September 9, 2015; Accepted with revision: March 2, 2016; Available online: March 24, 2016 http://dx.doi.org/10.5507/bp.2016.011

\begin{abstract}
${ }^{a}$ Department of Surgery I, Faculty of Medicine and Dentistry, Palacky University Olomouc, Czech Republic
${ }^{b}$ Department of Surgery I, University Hospital Olomouc, Czech Republic

'Department of Molecular Pathology, Faculty of Medicine and Dentistry, Palacky University Olomouc, Czech Republic ${ }^{d}$ Department of Medical Biophysics, Faculty of Medicine and Dentistry, Palacky University Olomouc, Czech Republic

eDepartment of Oncology, Faculty of Medicine and Dentistry, Palacky University Olomouc and University Hospital Olomouc, Czech Republic Corresponding author: Roman Havlik, e-mail: roman.havlik@fnol.cz
\end{abstract}

\section{INTRODUCTION}

The incidence of pancreatic cancer in the Czech Republic is rising. In Europe (2012), the highest world age-standardised incidence rates for pancreatic cancer are in the Czech Republic for both men (11.9/100000) and women (7.9/100000) (ref. $\left.{ }^{1-3}\right)$. The European Health Report (2012) named pancreatic cancer as the leading cause of cancer-related deaths in Europe. The prognosis for patients with pancreatic cancer is extremely poor, with the overall 5-year survival rate of only about $6 \%$ (ref. ${ }^{1,4}$ ). The diagnosis of pancreatic carcinoma at an early stage is uncommon. Radical resection is the only curative treatment modality that is possible in only $15-20 \%$ of cases. The National Cancer Database reported that nearly 38\% of radical resection candidates will not undergo surgery ${ }^{5}$. A quarter of patients have a locally advanced disease with approximately $53 \%$ of patients presenting with distant metastases. Several studies have analysed the prognostic factors of long-term survival and evaluated the 5-year survival in resected patients ${ }^{6-15}$ (Tables 1 and 2).
The aim of this study was to evaluate factors affecting the long-term survival (5-year survival) in a single tertiary centre; on a group of radically resected patients who had pancreatic cancer. A secondary aim was to identify the factors affecting their survival.

\section{METHODS}

\section{Selection of patients}

A database was prospectively maintained by the authors from January 2006. Patients who underwent surgery between January 2006 and December 2010 were evaluated in the present report. The main inclusion criteria included patients with pancreatic ductal adenocarcinoma (PDAC) on histopathological examination, and potentially curative resection (patients with $\mathrm{R} 2$ resections were not excluded). 7th edition UICC TNM classification (2011) was used to stage the tumours. All 90 consecutive patients underwent a standard pancreatic resection with a standard lymphadenectomy. Informed consent was waived as the study con- 
Table 1. Long term survival studies.

\begin{tabular}{|c|c|c|c|c|c|c|c|c|c|}
\hline Study & Period & No. & $\begin{array}{l}\text { PD } \\
(\%)\end{array}$ & $\begin{array}{l}\text { R0 } \\
(\%)\end{array}$ & $\begin{array}{l}\mathrm{R} 1 / 2 \\
(\%)\end{array}$ & $\begin{array}{l}\mathrm{N}+ \\
(\%)\end{array}$ & $\begin{array}{l}\text { VR } \\
(\%)\end{array}$ & $\begin{array}{l}\text { Median survival } \\
\text { (months) }\end{array}$ & $\begin{array}{c}\text { 5-yr survival, } \\
\mathrm{n}(\%)\end{array}$ \\
\hline Our study & $2006-2010$ & 90 & 83 & $\mathrm{nr}$ & $\mathrm{nr}$ & 51.6 & 4 & 17 & $17(18.9)$ \\
\hline Cleary (ref. ${ }^{6}$ ) & 1988-1996 & 123 & 91 & $\mathrm{nr}$ & 15 & 43 & 10 & 14 & $18(15)$ \\
\hline $\operatorname{Han}\left(\right.$ ref. $\left.^{7}\right)$ & 1985-1999 & 123 & 81 & 76 & 24 & 54 & 14 & 15 & $11(9)$ \\
\hline Howard $\left(\right.$ ref. $\left.^{8}\right)$ & $1990-2002$ & 226 & 90 & 70 & $28 / 2$ & 56 & 6 & 13 & $9(4)$ \\
\hline Cameron (ref. ${ }^{9}$ ) & $1969-2003$ & 405 & 100 & $\mathrm{nr}$ & $\mathrm{nr}$ & $\mathrm{nr}$ & $\mathrm{nr}$ & $\mathrm{nr}$ & $73(18)$ \\
\hline Ferrone $\left(\right.$ ref. $\left.{ }^{11}\right)$ & $1983-2001$ & 618 & 85 & 73 & $27 / 0$ & $\mathrm{nr}$ & 0 & $\mathrm{nr}$ & $75(12)$ \\
\hline Schnelldorfer (ref. ${ }^{12}$ ) & $1981-2001$ & 357 & 100 & 77 & $18 / 5$ & 49 & 13 & 17 & $62(17)$ \\
\hline Katz $\left(\right.$ ref. $\left.^{13}\right)$ & $1990-2002$ & 329 & 92 & 84 & $16 / 0$ & 48 & 33 & 24 & $88(27)$ \\
\hline Wentz (ref. ${ }^{14}$ ) & $1984-2009$ & 169 & $\mathrm{nr}$ & $\mathrm{nr}$ & $\mathrm{nr}$ & $\mathrm{nr}$ & $\mathrm{nr}$ & 15 & $46(27)$ \\
\hline Robinson (ref. ${ }^{24}$ ) & 2002-2009 & 134 & 100 & 32 & $68 / 0$ & 89.5 & $\mathrm{nr}$ & $\mathrm{nr}$ & $25(18.6)$ \\
\hline Kimura $\left(\right.$ ref. $^{15}$ ) & $1988-2008$ & 147 & 61 & $\mathrm{nr}$ & $\mathrm{nr}$ & $\mathrm{nr}$ & $\mathrm{nr}$ & $\mathrm{nr}$ & $18(12)$ \\
\hline Yamamoto (ref. ${ }^{4}$ ) & 2000-2008 & 96 & $\mathrm{nr}$ & $\mathrm{nr}$ & $\mathrm{nr}$ & $\mathrm{nr}$ & $\mathrm{nr}$ & 27 & $20(34)$ \\
\hline
\end{tabular}

PD - pancreatoduodenectomy, N+ - nodal positive status, VR - vascular resection, nr - not reported

Table 2. Factors affecting survival.

\begin{tabular}{|c|c|c|c|c|c|c|c|c|c|c|c|c|c|c|c|c|c|}
\hline Study & Size & Jaundice & N0 & LNR & $\mathrm{N}+$ & G & Stage & R0 & Comp & $\mathrm{CHT}$ ca & a $19-9$ & ALB & VI & PNI & PVI & NPAR & BT \\
\hline Our study & & & $\mathrm{u}$ & & & & $\mathrm{u}$ & & $\mathrm{m}$ & & & & $\mathrm{u}, \mathrm{m}$ & $\mathrm{u}$ & & & $\mathrm{u}$ \\
\hline Cleary (ref. ${ }^{6}$ ) & $\mathrm{u}$ & $\mathrm{u}$ & $\mathrm{u}$ & & & $\mathrm{u}, \mathrm{m}$ & $\mathrm{u}, \mathrm{m}$ & & & & & & & & & & \\
\hline Han (ref. $\left.{ }^{7}\right)$ & $\mathrm{u}$ & & $\mathrm{u}$ & & & & $\mathrm{u}, \mathrm{m}$ & $\mathrm{u}$ & & & & & & & & & \\
\hline Howard $\left(\right.$ ref. $\left.^{8}\right)$ & $\mathrm{m}$ & & & & & $\mathrm{m}$ & & $\mathrm{m}$ & $\mathrm{m}$ & & & & & & & & \\
\hline Ferrone (ref. ${ }^{11}$ ) & & & $\mathrm{m}$ & & & & $\mathrm{m}$ & & & & $\mathrm{u}, \mathrm{m}$ & & & & & & \\
\hline Schnelldorfer (ref. ${ }^{12}$ ) & $\mathrm{u}$ & & $\mathrm{u}, \mathrm{m}$ & & & & & & & & & $\mathrm{u}$ & & & & & \\
\hline Katz $\left(\right.$ ref. $\left.^{13}\right)$ & & & $\mathrm{u}, \mathrm{m}$ & $\mathrm{u}$ & & & & $\mathrm{u}$ & & & & & & & & $\mathrm{u}, \mathrm{m}$ & \\
\hline Hartwig (ref. ${ }^{22}$ ) & $\mathrm{u}$ & & $\mathrm{u}$ & $\mathrm{u}$ & & & & $\mathrm{u}$ & & & $\mathrm{u}$ & & & & & & \\
\hline Wentz $\left(\right.$ ref. $\left.^{14}\right)$ & & & $\mathrm{u}$ & $\mathrm{u}, \mathrm{m}$ & & $\mathrm{u}$ & & $\mathrm{u}$ & & $\mathrm{u}$ & $\mathrm{m}$ & & & & & & \\
\hline Robinson (ref. ${ }^{24}$ ) & & & $\mathrm{u}$ & $\mathrm{u}, \mathrm{m}$ & & & & & & & & & & $\mathrm{m}$ & & & \\
\hline Kimura (ref. ${ }^{15}$ ) & & & & & $\mathrm{u}, \mathrm{m}$ & & & $\mathrm{u}, \mathrm{m}$ & & & $\mathrm{u}$ & & & $\mathrm{u}, \mathrm{m}$ & & & \\
\hline Yamamoto (ref. $\left.{ }^{4}\right)$ & $\mathrm{u}, \mathrm{m}$ & & $\mathrm{u}$ & & & & $\mathrm{u}$ & $\mathrm{u}, \mathrm{m}$ & & & $\mathrm{u}$ & & & & $\mathrm{u}$ & & \\
\hline Strobel $\left(\right.$ ref. $\left.^{23}\right)$ & & & $\mathrm{u}$ & $\mathrm{u}$ & $\mathrm{u}$ & & & & & & & & & & & & \\
\hline
\end{tabular}

N0 - nodal negative status, LNR - lymph node ratio, N+ - nodal positive status, G - grade, R0 - radical resection, CHT - adjuvant chemotherapy, ALB - albumine, VI - vascular invasion, PNI - perineural invasion, PVI - portal vein infiltration, Comp - complications, NPAR - no previous attempt of resection, BT - blood transfusion, $\mathrm{u}$ - univariate analysis, $\mathrm{m}$ - multivariate analysis

ducted was a historic cohort. Factors analysed, included the age and gender of the patient, histopathological grading of the disease, histopathological evaluation including perineural invasion, vascular invasion and lymphatic invasion, lymph node infiltration, stage, postoperative complications with severity assessed according to ClavienDindo (CD) (ref. ${ }^{16}$ ), need for transfusion, redo-surgery and the administration of postoperative chemotherapy. Specific complications of pancreatic surgery were defined according to the ISGPS (International Study Group for Pancreatic Surgery) (ref. ${ }^{17-19}$ ). Adjuvant oncological therapy was indicated in accordance with the criteria and the recommendations of the Czech Oncological Society.

\section{Follow-up}

All patients were followed up by either the operating surgeon, oncologist or their GP. Data on all 90 patients were collected by direct contact with the patient or by telephone contact, with either their GP or a family member.
Patients surviving over 5 years were followed up personally by the operating surgeon.

\section{Statistical analysis}

Prospectively collected data was analysed with the statistical program, IBM SPSS Statistics version 22 (USA). The survival was evaluated using Kaplan-Meier method. The statistical significance of prognostic factors was assessed using the log-rank test and Cox regression analysis. $P$ values less than 0.05 were considered significant.

\section{RESULTS}

During the studied period, resections with curative intent were performed in 90 patients, 64 males $(71.1 \%)$ and 26 females $(28.9 \%)$. Mean age ( \pm standard deviation) was $63.2 \pm 8.6$ years. The median age of all patients was 63 years (range 40-81 years). Tumour localisation was within 
Table 3. Complications and reinterventions.

\begin{tabular}{|c|c|c|c|c|c|c|c|}
\hline & POPF & PPH & Wound & Abscess & DJAleak & RedoSurg & Total \\
\hline IIIa & 2 & & & 3 & & & 5 \\
\hline IIIb & 3 & 3 & 1 & & 1 & 8 & 8 \\
\hline IV & 1 & & & & & 1 & 1 \\
\hline V & 1 & & & & & 1 & 1 \\
\hline n 90 & $7.8 \%$ & $3.3 \%$ & $1.1 \%$ & $3.3 \%$ & $1.1 \%$ & $11.1 \%$ & $16.7 \%$ \\
\hline
\end{tabular}

POPF - pancreatic fistula, PPH - postpancreatectomy haemorrhage, DJA - duodenojejunalanastomosis

the head of pancreas in $76(84.5 \%)$ cases, body/tail in 11 (12.2\%) and multifocal in $3(3.3 \%)$ cases. Surgical procedures performed included pancreatic head resection in 75 (83.4\%) patients, total pancreatectomy in $4(4.4 \%)$, and distal pancreatectomy with splenectomy in $11(12.2 \%)$ patients. All these procedures were performed with a standard lymphadenectomy. A vascular (venous) resection was performed in 4 cases (4.4\%). Thirty-day mortality and in-hospital mortality occurred in 1 case $(1.1 \%)$, the 90 -day mortality was 3.3\%. Median survival was 16.9 months (95\%CI: 12.1 - 21.7). Median survival in the N0 group is 22.0 months $(95 \% \mathrm{CI}: 10.3$ - 33.7), median survival in the Npositive group is 13.3 months (95\%CI: 10.4 16.2). Forty-seven patients $(52.2 \%)$ did not experience any postoperative complications (CD 0), non-severe complications (CD I+II) were observed in twenty-seven (30\%), five patients $(5.6 \%)$ needed postoperative interventions without general anesthesia (CD IIIa) while ten (11.1\%) required a redo-surgery (CD IIIb-V), and one patient died (1.1\%, CD V).

Table 3 summarises types of postoperative complications encountered at different severity levels according to Clavien-Dindo classification, which required management. Clinically relevant postoperative pancreatic fistulas (POPF B,C) were the most common complications and all 7 patients $(7.8 \%)$ required re-intervention. A drainage procedure was needed in 3 patients and a salvage pancreatectomy was required in 4 patients with sepsis due POPF type C. Other interventions undertaken for the remaining complications included three $(3.3 \%)$ redo-surgeries for life-threatening postpancreatectomy haemorrhage $(\mathrm{PPH}$, all early, type B), re-suturing of insufficient duodenojejunal anastomosis $(1.1 \%)$, laparotomy re-suturing due its insufficiency in one patient $(1.1 \%)$ and CT drainage of peripancreatic abscesses in three (3.3\%). Patients with locally advanced tumours (pT3), with moderately and poorly differentiated tumours (G2-3), with or without lymph node involvement, perineural invasion, lymphatic invasion and vascular invasion were indicated for postoperative oncological therapy. Patients were in good general health (performance status 0-1 according to WHO), without significant weight loss. The provided treatment was either a modified DeGramont regimen of Leucovorin and 5-Fluorouracil, or Gemcitabine monotherapy. Treatment was initiated 4-7 weeks after the surgical procedure and lasted 6 months on average. Four to eight cycles of chemotherapy were administered. Relative contraindications for postoperative adjuvant therapy were comorbidity, poor nutritional status, age and patient's non-compliance. Information about oncological treatment were obtained in $80 \%$ patients. Adjuvant therapy following resection were administered in $75 \%$ of them (69.8\% gemcitabine, $9.4 \%$ fluoropyrimidine based, in $20.8 \%$ was not known).

Table 4. Univariate analysis of prognostic variables.

\begin{tabular}{|c|c|c|}
\hline Prognostic factors & $\begin{array}{c}\text { number of } \\
\text { patients }\end{array}$ & $\mathrm{P}$ \\
\hline Age & 89 & 0.518 \\
\hline$\leq 63$ & 45 & \\
\hline$>63$ & 44 & \\
\hline Gender & 89 & 0.303 \\
\hline female & 26 & \\
\hline male & 63 & \\
\hline Grade & 83 & 0.172 \\
\hline Well & 11 & \\
\hline Moderate & 34 & \\
\hline Poor & 38 & \\
\hline Perineural invasion & 84 & 0.022 \\
\hline $\mathrm{PN}+$ & 56 & \\
\hline PN- & 28 & \\
\hline Vascular invasion & 83 & 0.0003 \\
\hline $\mathrm{VI}+$ & 18 & \\
\hline VI- & 65 & \\
\hline Lymphatic invasion & 83 & 0.293 \\
\hline $\mathrm{LI}+$ & 23 & \\
\hline LI- & 60 & \\
\hline Lymph node infiltration & 89 & 0.001 \\
\hline No & 43 & \\
\hline $\mathrm{N}+$ & 46 & \\
\hline Stage & 89 & 0.013 \\
\hline Early stage & 42 & 0.003 \\
\hline Advanced stage & 47 & \\
\hline Complications Clavien-Dindo & 90 & 0.087 \\
\hline 0 & 47 & \\
\hline $\mathrm{I}-\mathrm{V}$ & 43 & \\
\hline Blood transfusion & 82 & 0.034 \\
\hline Yes & 54 & \\
\hline No & 28 & \\
\hline Redo surgery & 89 & 0.995 \\
\hline No & 79 & \\
\hline Yes & 10 & \\
\hline Chemotherapy & 71 & 0.538 \\
\hline yes & 53 & \\
\hline no & 18 & \\
\hline
\end{tabular}




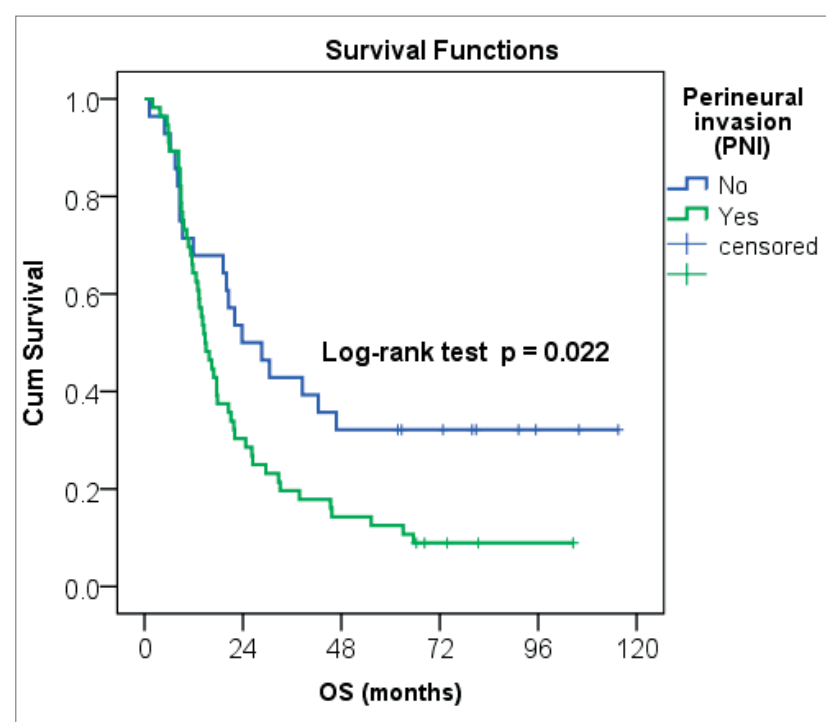

Fig. 1. Kaplan-Meier analysis - Perineural invasion (PNI).

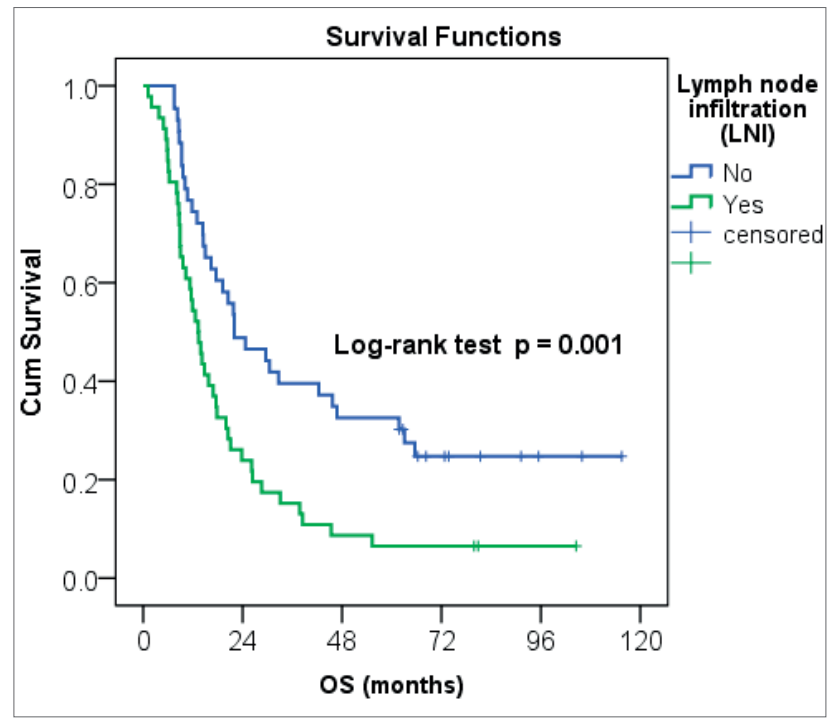

Fig. 3. Kaplan-Meier analysis - Lymph node infiltration (LNI).

\section{5-year survivors}

Seventeen patients (18.9\%) survived more than 5 years. The range of survival is $62-117$ months with median of 74 months. The five-year survival rate in the N0 subgroup was $32.6 \%$ (95\%CI: $18.6 \%$ - $46.6 \%$ ), range is $62-117$ months, median 68 months, while the five-year survival rate for the $\mathrm{N}+$ subgroup was only $6.5 \%$ (95\%CI: $0-13.7 \%$ ), the range is $81-106$ months, median 82 months. Of the group of 5-year survivors, $70.6 \%$ patients showed no evidence of recurrence. $29.4 \%$ experienced recurrent disease. The paraaortal lymph node metastases were diagnosed in two of these in the second year after surgery. Radio/chemotherapy in second line was successful in both. The total five-year relapse-free survival (RFS) rate is $15.6 \%$ (range is 14-117 months), with a mean of $66.0 \pm 28.8$ months and median of 67 months. Table 4 shows the analysis of the prognostic significance of studied factors. The univariate analysis confirmed that the absence of perineural invasion $(P=0.022)$ (Fig. 1$)$ and vascular invasion $(P=$

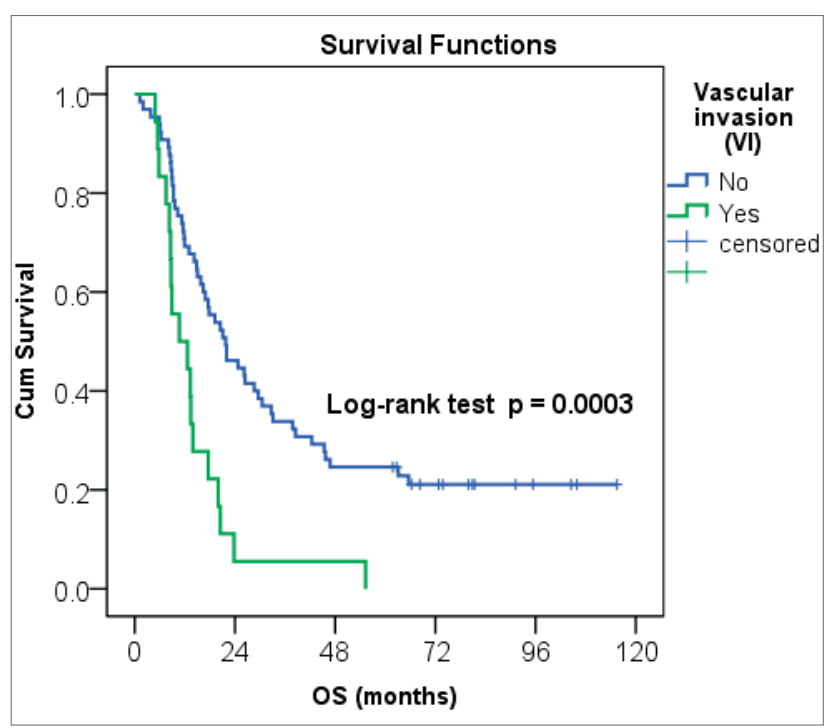

Fig. 2. Kaplan-Meier analysis - Vascular invasion (VI).

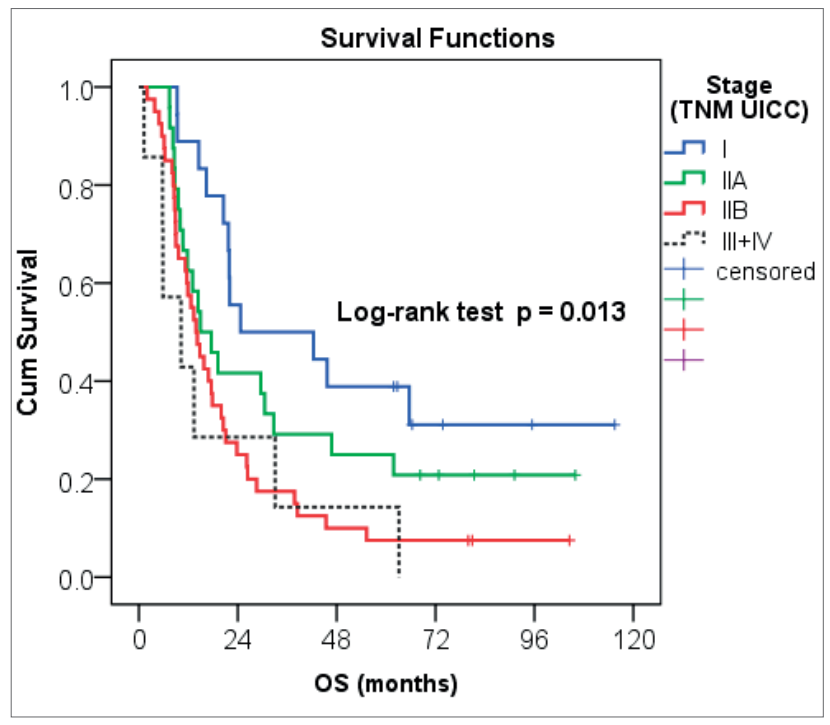

Fig. 4. Kaplan-Meier analysis - Stage (TNM UICC).

0.0003) (Fig. 2) in the histopathological examination of resected specimens, absence of lymph node infiltration $(P=0.001)$ (Fig. 3$)$, stage $(P=0.013)$ (Fig. 4), early stage (I,IIa vs. IIb,III,IV) $(P=0.003)$, and no blood transfusion $(P=0.034)$ were associated with longer overall survival. The operation time (median was $294 \mathrm{~min})(P=0.057)$ and complications free postoperative course (CD0) $(P=$ 0.087 ) were associated with longer overall survival, but were not statistically significant. On multivariate analysis vascular invasion $\mathrm{HR}=3.137$ (95\%CI: 1.692-5.816; $P$ $=0.0003)$ and postoperative complications $(\mathrm{I}-\mathrm{IV}) \mathrm{HR}=$ 2.004 (95\%CI: $1.198-3.354 ; P=0.008)$ were identified as significant independent predictors of survival.

\section{DISCUSSION}

PDAC has a dismal prognosis, even in those patients who are able to undergo radical resection. In the last 10 
years, there have been several studies evaluating 5-year survival and prognostic factors in pancreatic cancer patients after resection (Tables 1 and 2). The most frequently identified prognostic factors in these studies were N0 status and clear resection margins. The evaluation of resection margins has been modified according to the Leeds pathology protocol (LEEPP) (ref. ${ }^{20,21}$ ). The N-positive group can be stratified more accurately according to the number of positive nodes. The largest prospective study investigating these factors was conducted by the Heidelberg group. The Hartwig study 2011 (1071 pts/8 years), which evaluated prognostic factors and survival in patients undergoing resection for PDAC, identified tumour size, nodal status and distant metastases as independent predictors of patient survival. Additional prognostic parameters included age over 70, preoperative presence of IDDM, CA 19-9 over $400 \mathrm{U} / \mathrm{mL}$, negative resection margins according to the Leeds protocol, G1 grade and lymph node ratio (LNR) larger than 0.2. In the group of patients without the above-mentioned risk factors, 5-year survival was reached by more than $54 \%$; however, the overall 5-year survival rate (OS) was not reported ${ }^{22}$. Another prospective study ( 811 pts/12 years) by Strobel (2015), evaluated lymph nodes and the stratification of different prognoses according to the number of positive lymph nodes (PLN). The study demonstrated the importance of obtaining a high number of examined lymph nodes (ELN) (median 24, range 18-32), and the superiority of PLN compared to LNR in predicting survival. In the $\mathrm{N}$-positive group, median survival of patients with 1 PLN was comparable to the survival of N0 patients ${ }^{23}$. In this present analysis, demographic factors, tumour characteristics, stage, operative characteristics and postoperative therapy and their impact on long survival were studied. Age, gender, lymphatic invasion (LI), mild vs. severe complications, redosurgeries and postoperative chemotherapy were factors not affecting long term survival in this analysis. Operation time, absence of complications $(C D=0)$ and hospital stay affected long term survival, but were not statistically significant. On univariate analysis no vascular invasion (VI), no perineural invasion (PNI), no lymph node infiltration, stage, early stage I and IIa and no blood transfusion were associated with better long-term survival. On multivariate analysis, the independent favourable predictors of long survival were absence of vascular invasion and absence of postoperative complications. The vascular invasion in tumours was not studied in the cited cohorts presenting OS except in the Robinson's study; however, the authors were unable to prove that this factor had a statistically significant effect on the 5-year survival ${ }^{24}$. Other studies proved, in accordance with our study, that PNI and VI were associated with poor survival following pancreaticoduodenectomy ${ }^{25-27}$. Surprisingly, one study presented VI along with PNI to be an independent favourable prognostic factor in patients with locally advanced and metastatic pancreatic cancer ${ }^{28}$. In that large cohort the PNI, VI and LI was only detected in $7.2 \%, 6.1 \%$ and $3.3 \%$ respectively, contrarily to our study, where it was seen in $66.7 \%, 21.7 \%$ and $27.7 \%$. In our study only $\mathrm{N}$-positive/negative status was analysed. In the N0 subgroup, the 5-year survival rate was markedly higher than in the $\mathrm{N}$-positive group and more than three quarters of the 5-year survivors had a status of N0. Clinically relevant postoperative complications requiring re-interventions (CD IIIa-V) occurred in $16.7 \%$. Some studies considered severe complications CD IIIb-V. In this study, the rate of severe complications requiring redo-surgeries was $11.1 \%$. The most frequently encountered postoperative complications were clinically relevant POPF (46.7\%) and PPH (20\%). Redo-surgery rate was not evaluated in cited studies, in this study it did not affect survival. Compared with other studies, 5-year OS was $18.9 \%$, and a low 30 -day and in-hospital mortality rate $(1.1 \%)$ was observed. The median survival, however, was 16.9 months. Almost $65 \%$ of patients in our study died within 2 years of having surgery and this is comparable with a large study conducted in Johns Hopkins Hospital (2008). The latter showed a $40 \%$ survival rate in patients who had undergone adjuvant $\mathrm{CHT}$ as opposed to a $31 \%$ survival rate in those who were just observed within the 2 years after surgery ${ }^{29}$. Several RCT demonstrated improved survival with gemcitabine as adjuvant chemotherapy after radical surgery in PDAC patients ${ }^{30,31}$. Adjuvant chemotherapy was proven to be an independent prognostic factor of survival in the analysis of our previous smaller cohort ${ }^{30}$. In this extended cohort where data on postoperative chemotherapy was available ( $80 \%)$, postoperative chemotherapy was administered to $75 \%$ of them. More than two thirds $(69.8 \%)$ were treated with gemcitabine, $9.4 \%$ with fluoropyrimidine-based chemotherapy and in $20.8 \%$ the type of chemotherapy was not known. Chemotherapy in the presented cohort was not proven to be a significant factor affecting survival, probably due to the high rate of chemotherapy administration, but postoperative complications, in accordance with other studies, were associated with adjuvant therapy omission or delays ${ }^{29,32}$. Based on this analysis, several measures were taken. Resection margins (R0/R1) were not evaluated in this study, due to the absence of standard evaluation criteria during the studied period. The use of the Leeds Pathology Protocol (LEEPP), according to The Royal College of Pathologists, had not been implemented at the time of study and therefore was not used ${ }^{20,21}$. The Leeds protocol has now been implemented as a standard.

After analysing of postoperative complications, the technique used for pancreaticojejunal anastomosis has now been changed to a standardised duct-to-mucosa pancreaticojejunal anastomosis (PJA) in order to achieve a reduction in these PJA-related complications. Only a very small number of portal vein resections was performed (4.4\%) in this group and only one patient underwent neoadjuvant therapy. According to ISGPS and their criteria for borderline resectability, mesenterico-portal vein resections have been introduced into the surgical standard ${ }^{33}$. Neoadjuvant therapy is only administered in selected patients after multidisciplinary discussion; the protocol has not been yet standardised in our institution. 


\section{CONCLUSION}

Radical resection still represents the only potentially curative procedure in patients with PDAC. Surgical resection, when performed without complications and followed by adjuvant chemotherapy can lead to long-term survival. In the present cohort of patients with a potentially curative resection, $18.9 \%$ survived more than 5 years, and the 5-year relapse-free survival rate was $15.6 \%$. The rate of adjuvant oncological therapy was in $75 \%$ and most of them with gemcitabine (69.8\%). The absence of vascular invasion on the histopathological examination of the resected specimens and complication free postoperative course were identified as independent favourable prognostic factors.

\section{ABBREVIATIONS}

CD, Clavien Dindo system; CHT, Chemotherapy; CI, Confidence interval; CT, Computed tomography; ELN, Evaluated lymph node; GP, General practicioner; HR, Hazard ratio; IDDM, Insulin dependent diabetes mellitus; ISGPS, International study group for pancreatic surgery; LEEPP, Leeds pathology protocol; LI, Lymphatic invasion; LNR, Lymph node ratio; OS, Overall survival; PDAC, Pancreatic ductal adenocarcinoma; PJA, Pancreaticojejunal anastomosis; PLN, Positive lymph node; PNI, Perineural invasion; POPF, Postoperative pancreatic fistula; PPH, Postpancreatectomy haemorrhage; RFS, Relaps free survival; RCT, Randomized controlled trial; TNM, Tumor node metastasis classification; UICC, Union for international cancer control; VI, Vascular invasion.

Acknowlegement: The work was supported by grant IGA LF_2015_002.

Author contributions: ML: manuscript writing, literature search, database maintaining, surgical procedures, patients follow up; PS: literature search, surgical procedures, patients follow up; DK: manuscript preparation, surgical procedures; LB: database maintaining, literature search; CN: supervision; JE: pathological analysis, second examinations; JZ: statistical analysis; HS: patients follow up, administration of chemotherapy, manuscript correction; RV, MS: manuscript preparation, literature search; TY: manuscript preparation, translation, language corrections; RH: manuscript correction, supervision, surgical procedures.

Conflict of interest statement: The authors state that there are no conflicts of interest regarding the publication of this article.

\section{REFERENCES}

1. Euro Pancreatic Cancer Index 2014. Available from: http://www. healthpowerhouse.com

2. Ferlay J, Soerjomataram I, Ervik M, Dikshit R, Eser S, Mathers C, Rebelo M, GLOBOCAN 2012 v1.0, Cancer Incidence and Mortality Worldwide:
IARC 11 [Internet]. Lyon, France: International Agency for Research on Cancer; 2013. Available from: http://globocan.iarc.fr

3. Ferlay J, Steliarova-Foucher E, Lortet-Tieulent J, Rosso S, Coebergh JW, Comber H, Forman D, Bray F. Cancer incidence and mortality patterns in Europe: Estimates for 40 countries in 2012. European Journal of Cancer 2013;49(6):1374-403.

4. Yamamoto T, Yagi S, Kinoshita H, Sakamoto Y, Okada K, Uryuhara K, Morimoto T, Kaihara S, Hosotani R. Long-term survival after resection of pancreatic cancer: a single-center retrospective analysis. World J Gastroenterol 2015;21(1):262-8. doi: 10.3748/wjg.v21.i1.262

5. Bilimoria KY, Bentrem DJ, Ko CY, Stewart AK, Winchester DP, Talamonti MS. National failure to operate on early stage pancreatic cancer. Ann Surg 2007;246(2):173-80.

6. Cleary SP, Gryfe R, Guindi M, Greig P, Smith L, Mackenzie R, Strasberg S, Hanna S, Taylor B, Langer B, Gallinger S. Prognostic factors in resected pancreatic adenocarcinoma: analysis of actual 5-year survivors. J Am Coll Surg 2004;198:722-31.

7. Han SS, Jang JY, Kim SW, Kim WH, Lee KU, Park YH. Analysis of long-term survivors after surgical resection for pancreatic cancer. Pancreas 2006;32:271-5.

8. Howard TJ, Krug JE, Yu J, Zyromski NJ, Schmidt CM, Jacobson LE, Madura JA, Wiebke EA, Lillemoe KD. A margin-negative R0 resection accomplished with minimal postoperative complications is the surgeon's contribution to long-term survival in pancreatic cancer. J Gastrointest Surg 2006;10:1338-45.

9. Cameron JL, Riall TS, Coleman J, Belcher KA. One thousand consecutive pancreaticoduodenectomies. Ann Surg 2006;244(1):10-5.

10. Ferrone CR, Finkelstein DM, Thayer SP, Muzikansky A, FernandezdelCastillo C, Warshaw AL. Perioperative CA19-9 levels can predict stage and survival in patients with resectable pancreatic adenocarcinoma. J Clin Oncol 2006;24(18):2897-902.

11. Ferrone CR, Brennan MF, Gonen M, Coit DG, Fong Y, Chung S, Tang L, Klimstra D, Allen PJ. Pancreatic adeno-carcinoma: the actual 5-year survivors. J Gastrointest Surg 2008;12:701-6.

12. Schnelldorfer T, Ware AL, Sarr MG, Smyrk TC, Zhang L, Qin R, Gullerud RE, Donohue JH, Nagorney DM, Farnell MB. Long-term survival after pancreatoduodenectomy for pancreatic adenocarcinoma: is cure possible? Ann Surg 2008;247(3):456-62. doi: 10.1097/ SLA.0b013e3181613142

13. Katz MH, Wang $H$, Fleming JB, Sun $C C$, Hwang RF, Wolff RA, Varadhachary G, Abbruzzese JL, Crane CH, Krishnan S, Vauthey JN, Abdalla EK, Lee JE, Pisters PW, Evans DB Long-term survival after multidisciplinary management of resected pancreatic adenocarcinoma. Ann Surg Oncol 2009;16(4):836-47. doi: 10.1245/s10434-0080295-2

14. Wentz SC, Zhao ZG, Shyr Y, Shi CJ, Merchant NB, Washington K, Xia F, Chakravarthy AB. Lymph node ratio and preoperative CA 19-9 levels predict overall survival and recurrence-free survival in patients with resected pancreatic adenocarcinoma. World J Gastrointest Oncol 2012;4(10):207-15. doi: 10.4251/wjgo.v4.i10.207

15. Kimura K, Amano R, Nakata B, Yamazoe S, Hirata K, Murata A, Miura K, Nishio K, Hirakawa T, Ohira M, Hirakawa K. Clinical and pathological features of five-year survivors after pancreatectomy for pancreatic adenocarcinoma. World J Surg Oncol 2014;12:360. doi:10.1186/1477-7819-12-360

16. DeOliveira ML, Winter JM, Schafer M, Cunningham SC, Cameron JL, Yeo CJ, Clavien PA. Assessment of complications after pancreatic surgery: A novel grading system applied to 633 patients undergoing pancreaticoduodenectomy. Ann Surg 2006;244(6):931-9.

17. Bassi C, Dervenis C, Butturini G, Fingerhut A, Yeo C, Izbicki J, Neoptolemos J, Sarr M, Traverso W, Buchler M. Postoperative pancreatic fistula: an international study group (ISGPF) definition. Surgery 2005;138(1):8-13

18. Wente MN, Veit JA, Bassi C, Dervenis C, Fingerhut A, Gouma DJ, Izbicki JR, Neoptolemos JP, Padbury RT, Sarr MG, Yeo CJ, Büchler MW. Postpancreatectomy hemorrhage (PPH): an International Study Group of Pancreatic Surgery (ISGPS) definition. Surgery 2007;142(1):20-5.

19. Wente MN, Bassi C, Dervenis C, Fingerhut A, Gouma DJ, Izbicki JR, Neoptolemos JP, Padbury RT, Sarr MG, Traverso LW, Yeo CJ, Büchler MW. Delayed gastric emptying (DGE) after pancreatic surgery: a suggested definition by the International Study Group of Pancreatic Surgery (ISGPS). Surgery 2007;142(5):761-8

20. Verbeke CS, Menon KV. Redefining resection margin status in 
pancreatic cancer. HPB 2009;11(4):282-9. doi: 10.1111/j.14772574.2009.00055.x

21. The Royal College of Pathologists 2010. Standards and datasets for reporting cancers. Dataset for the histopathological reporting of carcinomas of the pancreas, ampulla of Vater and common bile duct May 2010, Available from: www.rcpath.org

22. Hartwig W, Hackert T, Hinz U, Gluth A, Bergmann F, Strobel O, Büchler MW, Werner J. Pancreatic cancer surgery in the new millennium: better prediction of outcome. Ann Surg 2011;254(2):311-9. doi: 10.1097/ SLA.0b013e31821fd334

23. Strobel O, Hinz U, Gluth A, Hank T, Hackert T, Bergmann F, Werner J, Büchler MW. Pancreatic adenocarcinoma: number of positive nodes allows to distinguish several N categories. Ann Surg 2015;261(5):9619. doi: 10.1097/SLA.0000000000000814

24. Robinson SM, Rahman A, Hauqk B, French JJ, Manas DM, Jaques BC Charnley RM, White SA. Metastatic lymph nide ration as an important prognostic factor in pancreatic ductale adenocrcinoma. Eur J Surg Oncol 2012;38(4):333-9.

25. Shimada K, Sakamoto Y, Sano T, Kosuge T. Prognostic factors after distal pancreatectomy with extended lymphadenectomy for invasive pancreatic adenocarcinoma of the body nd tail. Surgery 2006;139:288-95

26. Sierzega M, Popiela T, Kulig J, Nowak M. The ratio of metastatic/resected lymph nodes is an independent prognostic factor in patients with node-positive pancreatic head cancer. Pancreas 2006;33:240-5.

27. Garcea G, Dennison AR, Pattenden CJ, Neal CP, Sutton CD, Berry DP. Survival following curative resection for pancreatic ductal adenocarcinoma. A systematic review of the literature. JOP 2008;9:99-132.

28. Berardi R, Mandolesi A, Pellei C, Maccaroni E, Onofri A, Lucarell A, Biagetti S, Alfonsi S, Caramanti M, Savini A, Bearzi I, Cascinu S. Prognostic factors in pancreatic cancer: The role of perineural, vas- cular and lymphatic invasion and of Ca 19-9. J Gastroint Dig Syst 2013;3:134-8.

29. Wu W, He J, Cameron JL, Makary M, Soares K, Ahuja N, Rezaee N, Herman J, Zheng L, Laheru D, Choti MA, Hruban RH, Pawlik TM, Wolfgang CL, Weiss MJ. The impact of postoperative complications on the administration of adjuvant therapy following pancreaticoduodenectomy for adenocarcinoma. Ann Surg Oncol 2014;21:287381.

30. Lovecek M, Skalicky P, Klos D, Neoral C, Ehrmann J Jr, Zapletalova J, Svebisova H, Yogeswara T, Ghothim M, Vrba R, Havlik R. Surgical therapy of pancreatic cancer - 5 years survival. Rozhl Chir 2015;94:470-6.

31. Oettle H, Post S, Neuhaus P, Gellert K, Langrehr J, Ridwelsk K, Schramm H, Fahlke J, Zuelke C, Burkart C, Gutberlet K, Kettner E, Schmalenberg H, Weigang-Koehler K, Bechstein WO, Niedergethmann M, Schmidt-Wolf I, Roll L, Doerken B, Riess H. Adjuvant chemotherapy with gemcitabine vs. observation in patients undergoing curative-intent resection of pancreatic cancer: a randomized controlled trial. JAMA 2007;297:267-77.

32. Merkow RP, Bilimoria KY, Tomlinson JS, Paruch JL, Fleming JB, Talamonti MS, Ko CY, Bentrem DJ. Postoperative complications reduce adjuvant chemotherapy use in resectable pancreatic cancer. Ann Surg 2014;260:372-7.

33. Bockhorn M, Uzunoglu FG, Adham M, Imrie C, Milicevic M, Sandberg AA, Asbun HJ, Bassi C, Büchler M, Charnley RM, Conlon K, Cruz LF, Dervenis C, Fingerhutt A, Friess $H$, Gouma DJ, Hartwig W, Lillemoe KD, Montorsi M, Neoptolemos JP, Shrikhande SV, Takaori K, Traverso W, Vashist YK, Vollmer C, Yeo CJ, Izbicki JR; International Study Group of Pancreatic Surgery. Borderline resectable pancreatic cancer: A consensus statement by the International Study Group of Pancreatic Surgery (ISGPS). Surgery 2014;155:977-88. 